\title{
Nature of an Electric-Field-Induced Colloidal Martensitic Transition
}

\author{
Anand Yethiraj, ${ }^{1,2, *}$ Alan Wouterse, ${ }^{1,2}$ Benito Groh, ${ }^{3}$ and Alfons van Blaaderen ${ }^{1,2}$ \\ ${ }^{1}$ FOM Institute for Atomic and Molecular Physics, Kruislaan 407, 1098 SJ Amsterdam, The Netherlands \\ ${ }^{2}$ Soft Condensed Matter, Debye Institute, Utrecht University, Princetonplein 5, 3584 CC Utrecht, The Netherlands \\ ${ }^{3}$ Fachbereich Physik, Bergische Universität Wuppertal, D-42097 Wuppertal, Germany
}

(Received 17 April 2003; published 3 February 2004)

\begin{abstract}
We study the properties of a solid-solid close-packed to body-centered tetragonal transition in a colloidal suspension via fluorescence confocal laser scanning microscopy, in three dimensions and in real space. This structural transformation is driven by a subtle competition between gravitational and electric dipolar field energy, the latter being systematically varied via an external electric field. The transition threshold depends on the local depth in the colloidal sediment. Structures with order intermediate between close-packed and body-centered tetragonal were observed, with these intermediate structures also being stable and long lived. This is essentially a colloidal analogue of an "atomiclevel" interfacial structure. We find qualitative agreement with theory (based purely on energetics). Quantitative differences can be attributed to the importance of entropic effects.
\end{abstract}

DOI: 10.1103/PhysRevLett.92.058301

The solid state exhibits a rich variety of crystalline symmetries. Elemental solids are often body-centered cubic close to the melting point, and close packed at low temperatures. On the other hand, alloys of two or more elements display an astounding variety of crystal structures. Many solids undergo crystal-crystal transitions that can take place only under the change of an external parameter, with thermal fluctuations not being predominant. One such transition is a diffusionless solid-solid transformation known as a martensitic transition [1-4].

We report here on a 3D real-space study in a colloidal model system where we use an external electric field as a control parameter to study a martensitic crystal transformation in the presence of a second competing external field: gravity. Phenomenological modeling of martensitic transitions has indeed been shown to involve terms that act as effective long-range anisotropic interactions [5]. While the gravitational interaction is not relevant in atomic and molecular crystalline solids, it could well be that the complex phase behavior in alloys arises from a similar subtle competition of interactions. The only example we know where a colloidal model system was used to study a martensitic crystal transition focused on the effects of confining walls on crystal symmetry [6]. Brownian motion ensures that colloidal dispersions have a well-defined thermodynamic temperature and exhibit phases analogous to those seen in atomic systems; microscopy enables the quantitative study [7-9] of structure (including defects and grain boundaries) and dynamics on a single-particle level in the bulk: this is difficult in studies of atomic systems. In addition, controlled switching between two colloidal crystal structures can be used to make photonic band gap materials [10-12].

Our colloidal system is composed of silica spheres (radius, $R=692 \mathrm{~nm}$ ) suspended in a (20:80 volume ratio) water-dimethylsulfoxide (DMSO) liquid mixture, chosen
PACS numbers: 82.70.Dd, 05.70.Fh, 64.70.Kb, 83.80.Gv

to match the refractive index of the silica spheres. It has been previously established [13] that these spheres have a fluorescently labeled core (radius, $r=192 \mathrm{~nm}$ ) surrounded by a nonfluorescent shell (thickness $500 \mathrm{~nm}$ ), allowing three-dimensional sphere position determination without ambiguity even for touching spheres via fluorescence confocal laser scanning microscopy [7]. The gravitational height of our system, defined as the height that increases the gravitational potential energy by the thermal energy $k_{B} T$, is $h_{\text {grav }}=285 \mathrm{~nm}$. The colloids sediment towards the (unstructured) bottom surface and crystallize as close-packed hexagonal planes. The third hexagonal layer has two stacking possibilities: either similar as the first layer: $A B A$ [if repeated forming a hexagonal close-packed (HCP) crystal] or as $A B C$ [if repeated forming a face centered cubic (FCC) crystal]. Typical colloidal hard-sphere bulk crystals exhibit a random hexagonal close packing (RHCP) with, at high pressures, nearest-neighbor interparticle spacing $d \approx 2 R$. Surface groups on colloidal silica spheres ionize in aqueous suspension, giving rise to a surface charge and an electrostatic repulsive interparticle interaction. Hardsphere-like behavior is recovered with the addition of salt or impurities which ionize in aqueous solution: these ions are mobile and screen the electrostatic interaction and only the (entropic) excluded-volume interaction remains. In the absence of added salt the interactions in our colloidal system, while not strictly hard-sphere-like [nearest-neighbor interparticle spacing $d=(1.03) 2 R$ ], were consistent with hard-sphere-like behavior in the absence of an electric field [13].

The external electric field adds a long-range anisotropic dipolar interparticle interaction to the hard-sphere colloids (the "electrorheological effect" $[14,15]$ ), with an experimental equilibrium body-centered tetragonal (BCT: $a_{\mathrm{BCT}}=b_{\mathrm{BCT}}=\sqrt{3 / 2} c_{\mathrm{BCT}}$ and $c_{\mathrm{BCT}}=d=2 R$ ) 
crystal structure in a colloidal system [8] that agrees with dipolar energy calculations [16]. The $(1 \mathrm{MHz}$, ac) electric field is realized by applying a voltage between two $50 \mu \mathrm{m}$ alumel wires laid flat [see schematic Fig. 1(f)] between two glass plates. The space in between is filled with the colloidal suspension. The measurements are made in a central $0.1 \mathrm{~mm}$ region where the electric field is horizontal (pointing perpendicular to the gravitational field and to both the wires), uniform, and varies little with the $0.01 \mathrm{~mm}$ variation of height in the sediment. The field does not directly counteract sedimentation and the crystal can reorganize from $\mathrm{CP}$ to BCT stacking [Figs. 1(c) and 1(d)] with the hexagonal lattice planes moving apart by a fraction $(5 \%)$ of the lattice spacing $d$.

While the height change is small, the lateral displacement of the planes is larger $(0.29 d)$. The projection of two consecutive hexagonal layers in a CP stacking displays hexagonal symmetry, while that of two BCT-stacked layers displays distinct rectangular symmetry [Figs. 1(a) and $1(\mathrm{~b})]$. We quantify the symmetry change with a quasitwo-dimensional bond-order parameter [17,18]:
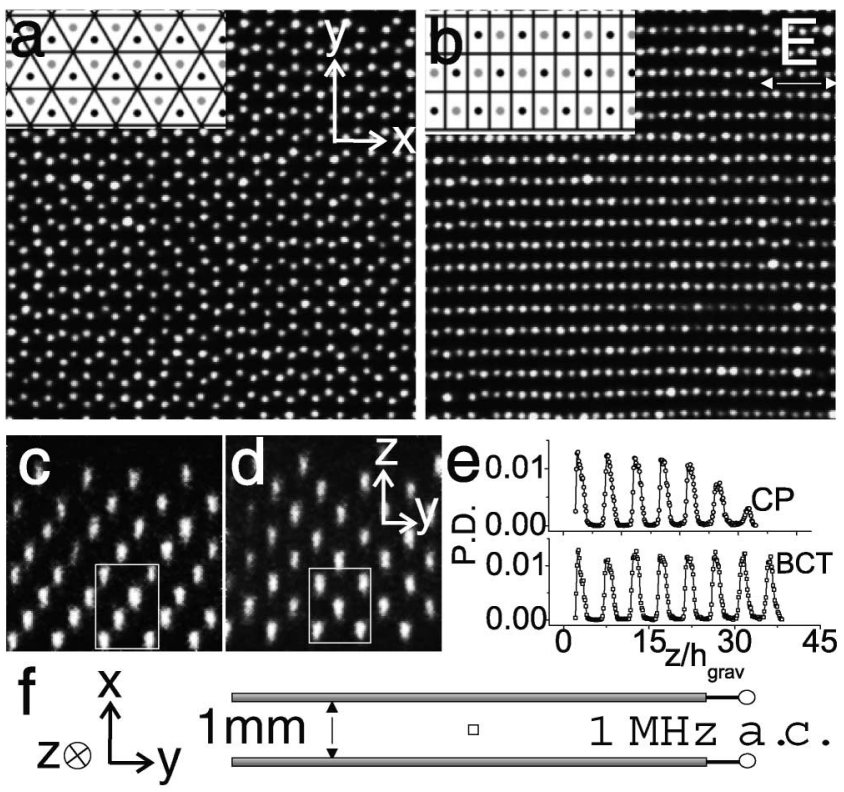

FIG. 1. Two-layer projections (TLP's) onto the $x y$ plane (field is along $x$, gravity along $z$ ) for colloids in (a) (low field) close packed (CP) and (b) (high field) body-centered tetragonal (BCT) crystal stackings. Only the (fluorescent) cores of the spheres is visible. Insets show TLP's for ideal lattices: gray and black correspond to different hexagonal planes, with spheres drawn smaller: at actual size adjacent gray (or black) spheres would touch. (c),(d) Snapshots in the $y z$ plane [same conditions as (a),(b), respectively]. (e) Particle-density profile P.D. (normalized to the total number of particles counted) for $\mathrm{CP}$ and BCT lattices, respectively. (f) Sample schematic: a $1 \mathrm{MHz}$ ac voltage is applied between two $50 \mu \mathrm{m}$ wires $1 \mathrm{~mm}$ apart (not drawn to scale), laid flat between two glass plates, and the space filled with colloidal suspension.

$$
\psi_{n}=\frac{1}{N}\left[\sum_{j=0}^{j=N-1} \ell_{j} \exp \left(i n \phi_{j}\right)\right] /\left[\sum_{j=0}^{j=N-1} \ell_{j}\right],
$$

where $n=4$ or 6 , and $N$ is the number of neighbors of the selected particle, and $\phi_{j}$ is the angle which the vector from the selected particle to the $j$ th neighbor makes with respect to the $x$ axis. When the $N$ neighbors are counted cyclically, $\ell_{j}$ is the length of the line segment joining two adjacent neighbors $j-1$ and $j$. The weighting with respect to the length ensured that an infinitesimal distortion from a perfect rectangular lattice gives rise to a continuous change in $\left|\psi_{4}\right|$. In experiments, thermal noise ensures finitely large distortions: our results are thus not affected by this choice of weighting.

The $\mathrm{CP}$ and the BCT two-layer projections (TLP) exhibit $\left|\psi_{6}\right|$ and $\left|\psi_{4}\right|$ values, respectively, that are close to unity (the maximum of the order parameter). In Figs. 1(a) and 1(b), we show an example of a CP and BCT-stacked TLP, respectively (model constructions in inset). Slices in the $y z$ plane [Figs. 1(c) and 1(d)] allow direct visualization of the stacking (FCC in this case and $\mathrm{BCT}$, respectively). Density profiles in the $z$ direction [Fig. 1(e)] demonstrate that the layering and hence the TLP is well defined. In the CP crystal, the colloidalcrystalline sediment is separated from the particle-free medium by a colloidal fluid region where the particle density decreases with height [13]. In contrast, the BCT is crystalline throughout and the layering more uniform; both these points are important for photonic applications.

The structural transformation proceeded continuously for each TLP, with intermediate lattice deformations being observed in a Voronoi color map [Fig. 2(a)]: each magenta cell is a distorted intermediate shape between rectangle and triangle. At low/high field strengths, the distribution of local $\left|\psi_{4}\right|$ values [Fig. 2(b)] is peaked at low/high $\left|\psi_{4}\right|$ values (corresponding to $\mathrm{CP} / \mathrm{BCT}$ ), respectively. However, for intermediate field strengths $\left(E_{\mathrm{rms}}=\right.$ $60 \mathrm{~V} / \mathrm{mm}$ for TLP3, 4 in a 14-layer sediment) there are patches of clearly $\mathrm{CP}$ and $\mathrm{BCT}$ regions separated by regions with distorted intermediate lattices. The distribution in this case is not bimodal (which would be expected for two-phase coexisting domains in the bulk) but flat, with a plateau at probability $p \approx 0.03$. The intersection of the low/high field distributions with $p \approx 0.03$ led to the coloring rules in Fig. 2(a).

In Fig. 2(c) the global bond-order parameter $\left\langle\left|\psi_{4}\right|\right\rangle$ is plotted as a function of electric field energy, for successive sets of TLP's in a 10-layer colloidal sediment (layer 10 corresponds to the top of the sediment). The threshold separating $\mathrm{CP}$ from $\mathrm{BCT}$ was determined from the dependence of $\left\langle\left|\psi_{4}\right|\right\rangle$ for artificial CP and BCT lattices on the mean-square displacement (MSD) from ideal lattice positions. The difference in $\left\langle\left|\psi_{4}\right|\right\rangle$ values, $\Delta\left\langle\left|\psi_{4}\right|\right\rangle$, of artificial CP and BCT lattices decreases from $\Delta\left\langle\left|\psi_{4}\right|\right\rangle=$ 1.0 for $\mathrm{MSD}=0$ to $\Delta\left\langle\left|\psi_{4}\right|\right\rangle=0.15$ for $\mathrm{MSD}=0.10 d$. In 

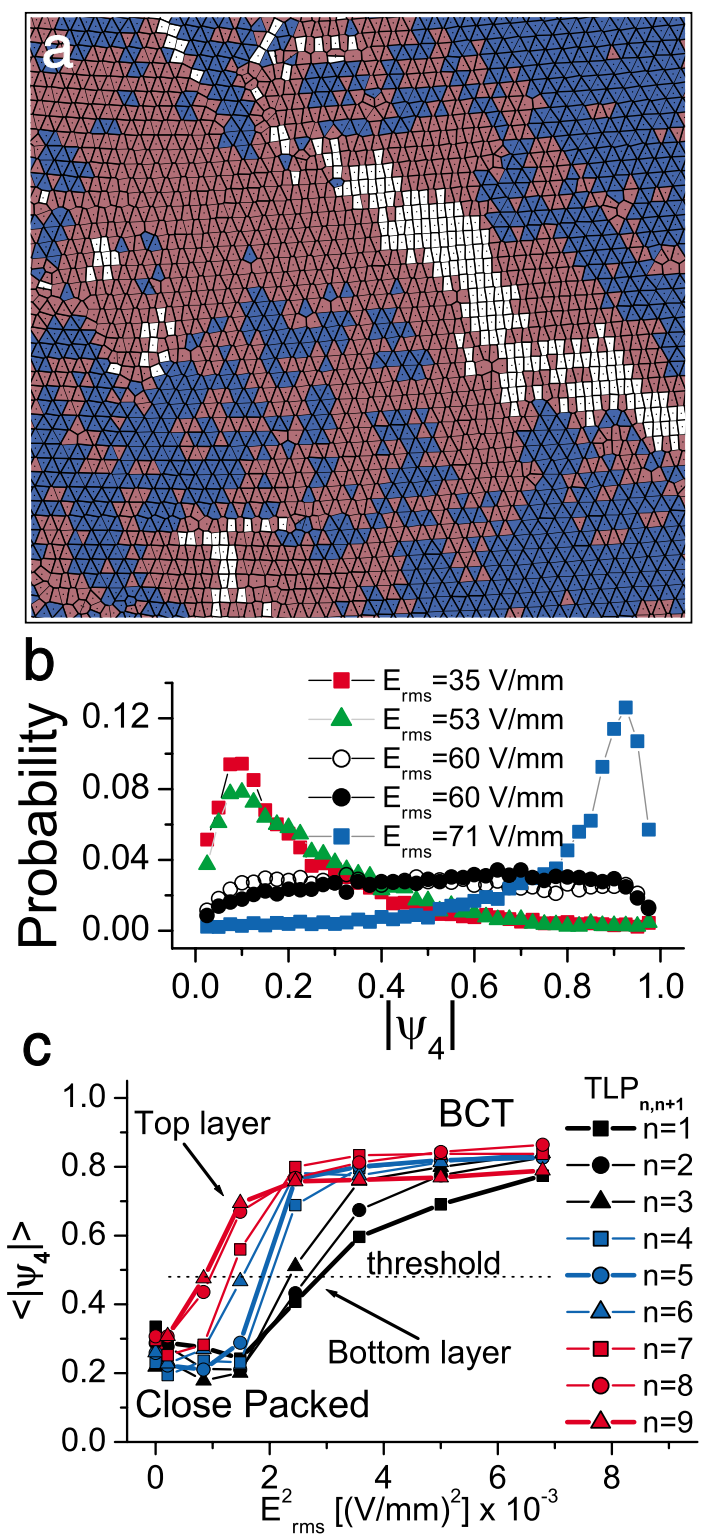

FIG. 2 (color). (a) Voronoi color map at solid-solid interface: $\mathrm{TLP}_{3,4}$ in a 14-layer sediment at $E_{\mathrm{rms}}=60.1 \mathrm{~V} / \mathrm{mm}$. Blue (dark gray), triangular cells correspond to $\left|\psi_{4}\right|<0.25$ (CP), white, rectangular cells to $\left|\psi_{4}\right|>0.8$ (BCT), and magenta (light gray) cells (distorted intermediate shapes between a rectangle and a triangle) to $0.25<\left|\psi_{4}\right|<0.8$. These intermediate structures are long lived (days). (b) Same sample as (a): $\operatorname{TLP}_{3,4}$. Distribution of $\left|\psi_{4}\right|$ values at low and high fields is peaked at $\left|\psi_{4}\right|=0.1$ and $\left|\psi_{4}\right|=0.9$, respectively. A flat distribution is seen at intermediate fields (not a double-peaked distribution, which would correspond to two-phase coexistence). (c) Bondorder parameter $\left\langle\left|\psi_{4}\right|\right\rangle$ vs $E_{\mathrm{rms}}^{2}$ for successive two-layer projections in a 10-layer sediment. The threshold of the CP-BCT transition is lowest for the top two-layer projection $\left(\mathrm{TLP}_{9,10}\right)$ and highest for the bottom $\left(\mathrm{TLP}_{1,2}\right)$.

the latter case, $\left\langle\left|\psi_{4}\right|\right\rangle_{\mathrm{CP}}=0.4$ and $\left\langle\left|\psi_{4}\right|\right\rangle_{\mathrm{BCT}}=0.55$. We defined the threshold to be the mean, $\left\langle\left|\psi_{4}\right|\right\rangle=0.48$. There was a clear transition from $\mathrm{CP}$ to $\mathrm{BCT}$ order as a function of increasing field energy for each pair of layers $\operatorname{TLP}_{n, n+1}$, with the threshold field strength increasing with decreasing layer number $n$.

The essential physics in this layer-by-layer transition is a subtle competition between gravity, which sets a depthdependent osmotic pressure, and the electric field, which sets the dipolar interaction energy. We compared these results quantitatively to an energy calculation for a tenlayer crystal where the stacking was varied parametrically between BCT and CP as a function of field energy. Perfectly ordered hexagonal layers of dipolar hard spheres were assumed. We employed the expression

$$
U=2 \pi \frac{2}{\sqrt{3}} \frac{p^{2}}{d^{2}} \sum_{\mathbf{G} \neq 0} \frac{G_{x}^{2}}{G} \cos \left(x G_{x}+y G_{y}\right) \exp (-G z)
$$

for the energy of a dipole at point $(x, y, z)$ above a layer. The sum runs over the vectors $\mathbf{G}$ of the reciprocal twodimensional lattice, $p$ is the dipole moment, the dipoles point into the $x$ direction and one lattice site is at the origin. This expression is derived by calculating the electrostatic potential of two slightly shifted opposite point charge lattices. Using Eq. (2) the total dipolar interaction energy is obtained by summing over the different layers. The total dipole plus gravitational energy was minimized with respect to the relative horizontal shifts of the layers, varying the vertical shift so that the spheres of diameter $d=2 R$ stay in contact. It is found that, starting from the $\mathrm{CP}$ configuration, the layers shift only perpendicularly to the dipole direction. From the layer positions, we then computed the $\left\langle\left|\psi_{4}\right|\right\rangle$ of successive TLP's as a function of field energy [Fig. 3(a)]. Screening, if modeled by an effective dielectric constant, would affect only the prefactor in Eq. (2). We normalize thresholds using the absolute value of the topmost layer threshold as a reference. The threshold energy is found theoretically to increase linearly with decreasing TLP number (increasing depth in the sample), while experimentally the dependence is sublinear [Fig. 3(b)], gray circles and black squares, respectively). On rescaling the field energies with respect to these thresholds [Fig. 3(c)] the theoretical curves fall on a single scaling curve. The experimental ones do not. The bottom layer looks clearly different from the rest and is traceable to poor hexagonal $\left(\left|\psi_{6}\right|\right)$ order, caused by sticking of some spheres at the silica substrate. The remaining experimental curves all have high and comparable $\left|\psi_{6}\right|$, and their nonrescalability is not traceable to this artifact. It is therefore a genuine effect and probably arises from neglected entropic effects.

Colloids provide an interesting model system to study (martensitic) solid-solid transitions. We observed a height-dependent CP to BCT phase transition, in qualitative agreement with an energy calculation; quantitative deviation could arise from entropic effects which are clearly important because we observed significant Brownian motion. The experimental system is also richer in other ways. The observation of patches of CP and BCT 


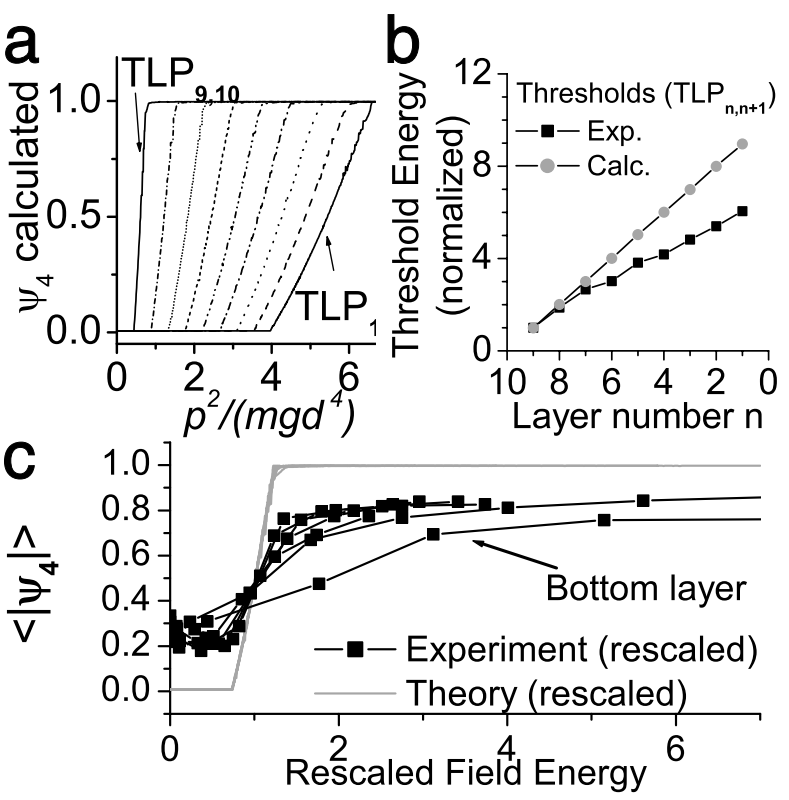

FIG. 3. (a) Theoretical values of $\left|\psi_{4}\right|$ vs reduced dipolar energy for a 10-layer sediment of dipolar spheres with dipole moment $p$, mass $m$, and diameter $d$ in the presence of gravity. $\mathrm{TLP}_{1,2}$ and $\mathrm{TLP}_{9,10}$ correspond to two-layer projections of the bottom and the top two layers, respectively. (b) Experimental and theoretical dipolar energy thresholds (both normalized with respect to the corresponding threshold for the topmost TLP). (c) Theoretical curves for $\left|\psi_{4}\right|$ vs dipolar energy collapse onto one curve, when rescaled with respect to the thresholds in (b), but experimental ones do not.

at the transition threshold suggests two-phase coexistence which can imply a first-order nucleation and growth mechanism. But an incomplete top layer (present in our experiments) gives rise to a spatially varying osmotic pressure and long-time measurements over several hours indicated that the apparently coexisting domains ("patches") were stationary (not expected with true coexistence domains). In fact, a close-packed patch in a BCT TLP was always correlated with an incomplete top layer (essentially a "step-edge" at the interface).

The observation of intermediate configurations in between the initial and final structures close to the transition threshold could, on the other hand, imply a transition where the energy barrier between the two phases is small compared to $k_{B} T$. However, these structures are observed only near the solid-solid interface and not in the bulk. It is apparent that we are observing the colloidal analog of an "atomic-level" interface across which, even at a firstorder transition (such as the gas-liquid transition), the order parameter would vary continuously. We can thus not use this to comment on phase transition order. This system will allow us in the future to address questions of interface kinetics on the single-particle level.

The martensitic crystal-crystal transition as a function of an external field also provides a novel way to sensitively control colloidal-crystalline structures as well as enhance long-range order, with applications in photonic band gap crystals that are currently being pursued.

We acknowledge useful discussions with J. Thijssen, C. Das, and M. Wortis; C. Wisman for help with the electronics; J. Hoogenboom for the particle synthesis; and B. Mulder and J. H. for a critical reading of the manuscript. This work is part of the research program of the "Stichting voor Fundamenteel Onderzoek der Materie (FOM)," which is financially supported by the "Nederlandse organisatie voor Wetenschappelijke Onderzoek (NWO)."

*Present address: Chemistry Department, University of British Columbia, 2036 Main Mall, Vancouver, British Columbia, V6T1Z1, Canada.

[1] A. G. Khachaturyan, Theory of Structural Transformations in Solids (John Wiley \& Sons, New York, 1983).

[2] S. Kartha et al., Phys. Rev. Lett. 67, 3630 (1991).

[3] M. Rao and S. Sengupta, Phys. Rev. Lett. 78, 2168 (1997).

[4] F. J. Perez-Reche et al., Phys. Rev. Lett. 87, 195701 (2001).

[5] W. C. Kerr et al., Phase Transit. 69, 247 (1999).

[6] J. A. Weiss et al.,J. Chem. Phys. 103, 1180 (1995).

[7] A. van Blaaderen and P. Wiltzius, Science 270, 1177 (1995).

[8] U. Dassanayake, S. Fraden, and A. van Blaaderen, J. Chem. Phys. 112, 3851 (2000).

[9] A. Yethiraj and A. van Blaaderen, Nature (London) 421, 513 (2003).

[10] K. M. Ho, C. T. Chan, and C. M. Soukoulis, Phys. Rev. Lett. 65, 3152 (1990).

[11] R. M. Amos et al., Electron. Lett. 36, 1411 (2000).

[12] A. van Blaaderen et al., in Photonic Crystals and Light Localization in the 21st Century, edited by C.M. Soukoulis, NATO Advanced Study Institute (Kluwer Academic, Dordrecht, 2001), p. 239.

[13] J. P. Hoogenboom et al., J. Chem. Phys. 117, 11320 (2002).

[14] J. E. Martin, J. Odinek, and T. C. Halsey, Phys. Rev. Lett. 69, 1524 (1992).

[15] M. Parthasarathy and D. J. Klingenberg, Mater. Sci. Eng. R17, 57 (1996).

[16] R. Tao and J. M. Sun, Phys. Rev. Lett. 67, 398 (1991).

[17] P. J. Steinhardt, D. R. Nelson, and M. Ronchetti, Phys. Rev. B 28, 784 (1983)

[18] C. A. Murray, in Bond-Orientational Order in Condensed Matter Systems, edited by K. J. Strandburg (Springer, Berlin, 1991). 